\title{
A SURVEY ON THE GEOMETRY OF ISOMETRIC ACTIONS
}

\section{Claudio Gorodski}

\begin{abstract}
We survey on our results on the geometry of the orbits of isometric actions of compact Lie groups on complete Riemannian manifolds.
\end{abstract}

\section{Introduction}

In this short survey article, we attempt to present our results about some of the most important classes of isometric actions of compact Lie groups on complete Riemannian manifolds. In doing so, we tried to put the material in a historical perspective. As a matter of fact, most of the aspects of the subjects covered here originated in the works of Chern-Lashof and Bott-Samelson in the late fifties, but other topics are older, have evolved quite independently, and have only later merged together into the same subject.

We start with the results of Bott-Samelson on variationally complete actions, and we explain their relation to focal point theory. Then we move on to the work of Conlon and Dadok on polar and hyperpolar actions, and we discuss recent results on the subject. Then we go back to the Chern-Lashof theory and Kuiper's reformulation in terms of critical point theory. This serves as a prelude for the next topic, which is taut submanifolds. Here we discuss equivalent formulations of the notion of tautness following Banchoff and Carter-West, and we present a list of examples and classification results. Finally, we introduce the notion of copolarity of isometric actions. A detailed and updated, but by no means complete, bibliography is appended at the end. For further details and results, see [CR85, Cec97, Tho98, Tho00, Gor01, BCO03]. 


\section{Variational completeness}

In 1958, Bott and Samelson ([BS58]; see also [Bot56]) introduced the concept of variational completeness for isometric group actions and developed powerful Morse theoretic arguments to compute the homology and cohomology of orbits of variationally complete actions. We will review their concept in a moment. As a motivation, we first mention that, roughly speaking, an isometric action of a compact Lie group on a complete Riemannian manifold is variationally complete means if it produces enough Jacobi fields along geodesics to determine the multiplicities of focal points to the orbits.

More precisely, let $N$ be a submanifold of a complete Riemannian manifold $M$. Let $\eta: \nu(N) \rightarrow M$ denote the endpoint map of $N$, that is, the restriction of the exponential map of $M$ to the normal bundle of $N$. A point $q=\eta(v)$ is a focal point of $N$ in the direction of $v \in \nu(N)$ of multiplicity $m>0$ if $d \eta_{v}: T_{v} \nu(N) \rightarrow T_{q} M$ is not injective and the dimension of its kernel is $m$. Let $v \in \nu_{p}(N)$, and let $\gamma_{v}$ denote the geodesic $t \mapsto \exp _{p}(t v)$. A Jacobi field along $\gamma_{v}$ is called an $N$-Jacobi field if it is the variational vector field of a variation through geodesics that are at time zero orthogonal to $N$. We will denote the space of $N$-Jacobi fields along $\gamma_{v}$ by $\mathcal{J}^{N}\left(\gamma_{v}\right)$. It is not difficult to see that $J$ is an $N$-Jacobi field along $\gamma_{v}$ if and only if $J(0) \in T_{p} N$ and $J^{\prime}(0)+A_{v} u \in \nu_{p}(N)$, where $p$ is the footpoint of $v, u=J(0)$ and $A_{v}$ is the Weingarten map in the direction $v$. The point $q$ is a focal point of $N$ in the direction $v$ if there is an $N$-Jacobi field along $\gamma_{v}$ that vanishes at $q$. We will denote the space of $N$-Jacobi fields along $\gamma_{v}$ that vanish at $q$ by $\mathcal{J}_{q}^{N}\left(\gamma_{v}\right)$. Now an isometric action of a compact Lie group $G$ on the complete Riemannian manifold $M$ is called variationally complete if every Jacobi field $J \in \mathcal{J}_{q}^{N}\left(\gamma_{v}\right)$, where $N$ is a $G$-orbit and $q$ is a focal point of $N$ in the direction of $v$, is the restriction along $\gamma_{v}$ of a Killing field on $M$ induced by the action of $G$.

Let $G / K$ be a compact symmetric space. Bott and Samelson proved in [BS58] that the action of $K$ on $G / K$ by left multiplication is variationally complete and so is the action of $K \times K$ on $G$ by left and right multiplication. This was gener- 
alized by Hermann in [Her60], who showed that if $K_{1}$ and $K_{2}$ are two symmetric subgroups of the same compact Lie group $G$, then the action of $K_{1}$ on $G / K_{2}$ is variationally complete and so is the action of $K_{1} \times K_{2}$ on $G$. It was also proved in $[\mathrm{BS} 58]$ that the linear isotropy representation of the symmetric space $G / K$ is variationally complete. In the same paper, Bott and Samelson constructed an explicit $\mathbf{Z}_{2}$-homology basis for the orbits of variationally complete actions.

\section{$3 \quad$ Polar and hyperpolar actions}

In 1971, Conlon [Con71] considered actions of a Lie group $G$ on a complete Riemannian manifold $M$ with the property that there is a connected, complete submanifold $\Sigma$ of $M$ that meets all orbits of $G$ in such a way that the intersections between $\Sigma$ and the orbits of $G$ are all orthogonal. Such a submanifold is called a section and an action admitting a section is called polar. It is easy to see that a section $\Sigma$ is totally geodesic in $M$. An action admitting a section that is flat in the induced metric is called hyperpolar. The main theorem in [Con71] states that a hyperpolar action of a compact Lie group on a complete Riemannian manifold is variationally complete. Notice that Conlon does not assume in [Con71] that $\Sigma$ is properly embedded, although this has been required in some of the later literature on the subject. On the other hand, he did assume that $\Sigma$ is closed, but this is not necessary for the proof of his main theorem. We also remark that it is not even necessary to assume that $\Sigma$ is complete, as this has been shown to be a consequence of the definition by Boualem [Bou95] and later, independently, by Heintze, Liu and Olmos [HLO00].

In the case of Euclidean spaces, there is clearly no difference between polar and hyperpolar representations since totally geodesic submanifolds of an Euclidean space are affine subspaces. Moreover, the question whether $\Sigma$ should be required to be properly embedded or not becomes redundant. It follows from É. Cartan's theory of symmetric spaces that the linear isotropy representations of the symmetric spaces are polar representations. Conversely, it turns out that a polar representation is orbit equivalent to the isotropy representation of a 
symmetric space. (We recall that two actions are said to be orbit equivalent if there is an isometry between the action spaces under which the orbits of two actions correspond.) This follows from the classification of polar representations by Dadok [Dad85]. Lists of all polar representations can be found in [EH99] (irreducible case) and in [Ber01] (reducible case); see also [GT00] (both cases). Di Scala and Olmos gave in [DO01] a short proof that a variationally complete representation of a compact Lie group is polar.

Next we discuss the case of compact symmetric spaces. There are two important classes of examples of hyperpolar actions on them. It is clear that a cohomogeneity one action of a compact Lie group on a compact symmetric space is hyperpolar. Besides that, the Hermann examples referred to above turn out to be hyperpolar actions. Kollross [Kol02] classified hyperpolar actions on compact irreducible symmetric spaces. It follows from his classification that, in the irreducible case, all hyperpolar actions belong to either one of these classes. Thorbergsson and I proved in [GT02b] that a variationally complete action on a compact symmetric space is hyperpolar. The idea of this proof is to lift the action to a variationally complete isometric action on an appropriate infinite dimensional Hilbert space which submerges onto the symmetric space, and to use an argument similar to the argument used in [DO01] for the finite dimensional linear case.

It is easy to construct examples of polar actions which are not hyperpolar on compact symmetric spaces of rank one as can be seen from the classification of polar actions on such spaces in [PT99]. So far no example of a polar action which is not hyperpolar on an irreducible symmetric space of rank greater than one has been found. It has been shown that the classes of polar and hyperpolar actions coincide in the compact irreducible Hermitian symmetric spaces ([PT02, BG05, Bil04]). The question whether the two classes of actions must coincide in any irreducible symmetric space of rank greater than one remains a major open problem in the area. ${ }^{1}$ In [Gor04a], a simple criterium for a closed subgroup

\footnotetext{
${ }^{1}$ Added in Proof. On February 18th, 2005, A. Kollross announced in the Oberseminar Differentialgeometrie in the University of Augsburg a complete classification of polar actions
} 
of isometries of a compact symmetric space to act polarly was devised. This might be helpful when dealing with the above problem. In the same paper, as an application, it was shown that a polar action on a compact symmetric space admitting a totally geodesic principal orbit must be the standard action of an orthogonal group (of any rank) on a sphere.

\section{The Chern-Lashof theorem}

For an isometric immersion $f$ of a compact manifold $M$ into an Euclidean space $\mathbf{R}^{m}$, Chern and Lashof [CL57] introduced the total absolute curvature $\tau(f)$ as the normalized volume of the unit normal bundle with respect to the Gauss map. Namely,

$$
\tau(f)=\frac{1}{\operatorname{vol}\left(S^{m-1}\right)} \int_{\nu^{1}(M)}|G| d \operatorname{vol}_{\nu^{1}(M)},
$$

where $\eta^{*} d \mathrm{vol}_{S^{m-1}}=G d \mathrm{vol}_{\nu^{1}(M)}$, and $\eta: \nu^{1}(M) \rightarrow S^{m-1}$ is the Gauss map. They proved that $\tau(f)$ is bounded below by the Morse number $\gamma(M)$, which is the minimum number of critical points which any Morse function on $M$ can have. Recall that the Morse inequalities say that $\gamma(M) \geq \beta(M ; \mathbf{F})$, where $\beta(M ; \mathbf{F})$ is the sum of the Betti numbers of $M$ with respect to the field $\mathbf{F}$. An immersion $f$ which attains this lower bound is said to have minimum total absolute curvature. Chern and Lashof also proved that if $\tau(f)=2$, then $M$ is a convex hypersurface in an affine subspace.

Kuiper reformulated these results in terms of critical point theory. In [Kui58], he showed that the infimum of the total absolute curvature $\tau(f)$ over all immersions of $M$ into all Euclidean spaces is the Morse number $\gamma(M)$. In [Kui62], he introduced a concept of generalized convexity in terms of intersections with half-spaces and injectivity of induced maps on homology. Note that the designation "tight" in this context was first used by Banchoff in [Ban65] in conjunction with his introduction of the two-piece property. An immersion $f$ of a compact

on compact symmetric spaces of rank greater than one with simple group of isometries, whose main consequence, according to him, is the coincidence of the classes of polar and hyperpolar actions on those spaces. 
manifold $M$ into an Euclidean space is said to be tight with respect to the field of coefficients $\mathbf{F}$ (or, for short, $\mathbf{F}$-tight) if the induced homomorphism

$$
H_{*}\left(f^{-1} \mathcal{H} ; \mathbf{F}\right) \rightarrow H_{*}(M ; \mathbf{F})
$$

in singular homology is injective for almost every closed half-space $\mathcal{H}$ in the ambient Euclidean space, whereas $f$ is said to have the two-piece property (TPP) if $f^{-1} \mathcal{H}$ is connected for every closed half-space $\mathcal{H}$ in the ambient Euclidean space. It can be easily shown that in both of these definitions we need only to consider half-spaces $\mathcal{H}$ which are defined by height functions that restrict to Morse functions on $M$. Plainly, then, we see that every tight immersion has the TPP. It is also interesting to notice that these properties are invariant under projective transformations, in the sense that one adds a hyperplane at infinity and considers images of submanifolds under projective transformations that do not meet the hyperplane at infinity.

An equivalent definition of $\mathbf{F}$-tightness for an immersion $f: M \rightarrow \mathbf{R}^{m}$ is requiring that every height function $h_{\xi}(x)=\langle f(x), \xi\rangle, x \in M$, which is a Morse function, has the property that its number of critical points is equal to the sum of the Betti numbers of $M$ relative to $\mathbf{F}$, i. e. $h_{\xi}$ is $\mathbf{F}$-perfect. It follows that a F-tight immersion of a compact manifold has minimum total absolute curvature, since total absolute curvature is the mean number of critical points of height functions on $M$. Note that in this case we also have that the Morse number $\gamma(M)$ equals the sum of the Betti numbers of $M$ relative to $\mathbf{F}$.

An important observation of Kuiper regarding the codimension of substantial tight immersions into Euclidean spaces appeared already in his first papers [Kui58, Kui61] on the subject: a substantial immersion $f$ of a compact $n$-dimensional manifold that satisfies the TPP admits a point where the second osculating space coincides with the ambient space. (We recall that an immersion $f: M \rightarrow \mathbf{R}^{m}$ is called substantial if its image does not lie in any affine hyperplane of $\mathbf{R}^{m}$.) Here the second osculating space of $f$ at $p$ is the affine space spanned by the first and second partial derivatives of $f$ at $p$. Counting these derivatives shows that the dimension of the second osculating space can 
be at most $\frac{1}{2} n(n+3)$. Therefore the codimension of the immersion can be at most $\frac{1}{2} n(n+1)$. The Veronese embedding of the real projective space $P^{n}(\mathbf{R})$ is tight in $\mathbf{R}^{\frac{1}{2} n(n+3)}$ showing that this estimate is optimal.

\section{Taut submanifolds}

The beginnings of the study of taut immersions can be traced back to Banchoff's paper [Ban70] where he attempted to classify tight surfaces which lie in a Euclidean sphere $S^{m-1} \subset \mathbf{R}^{m}$. In this case, we remark that the critical point theory of height functions is the same as that of distance functions $L_{q}(x)=\|f(x)-q\|^{2}, q \in \mathbf{R}^{m}$. Carter and West defined in [CW72] an immersion $f$ of a compact manifold to be taut with respect to the field $\mathbf{F}$ (or $\mathbf{F}$-taut, for short) if every Morse distance function $L_{q}$ has the minimum number of critical points allowed by the Morse inequalities with respect to $\mathbf{F}$. It follows from the preceeding remark that a tight spherical immersion is taut. Also, we observe that a taut immersion $f$ must be an embedding, for if $q$ is was double point in the image then the distance function $L_{q}$ would have two minima and one could then perturb $q$, if necessary, in order to obtain a Morse distance function with two local minima. Moreover, as was done for tightness, one sees that a submanifold $M \subset \mathbf{R}^{m}$ is $\mathbf{F}$-taut if and only if the induced homomorphism

$$
H_{*}(M \cap B ; \mathbf{F}) \rightarrow H_{*}(M ; \mathbf{F})
$$

in singular homology is injective for almost every closed ball $B$ in $\mathbf{R}^{m}$. It is then clear that tautness is conformally invariant. Furthermore, one sees that a taut submanifold $M$ is tight, because for any half-space $\mathcal{H}$ defined by a Morse height function one can construct a closed ball $B$ such that $M \cap \mathcal{H}$ is a strong deformation retract of $M \cap B$.

We next give some examples of tautly embedded submanifolds. The Clifford tori $S^{n_{1}}\left(r_{1}\right) \times \cdots \times S^{n_{k}}\left(r_{k}\right) \subset S^{n_{1}+\ldots+n_{k}}(1)$ where $r_{1}^{2}+\ldots+r_{k}^{2}=1$, and the standard embeddings of the projective spaces $P^{n}(\mathbf{F}), \mathbf{F}=\mathbf{R}, \mathbf{C}, \mathbf{H}, \mathbf{O}$ are taut, since these are tight spherical embeddings. In the case of spheres, a substantial 
taut embedding of a sphere must be spherical and of codimension one. (In fact, such an $f: S^{n} \rightarrow \mathbf{R}^{m}$ is tight, whence $m=n+1$ and $f\left(S^{n}\right)$ is a convex hypersurface by the Chern-Lashof theorem. Now stereographic projection maps $f\left(S^{n}\right)$ into a taut submanifold of $\mathbf{R}^{n+2}$ which cannot be substantial, again by the Chern-Lashof theorem. Therefore, we see that $f\left(S^{n}\right)$ is spherical.) If $M$ is an $n$-dimensional taut hypersurface in $\mathbf{R}^{n+1}$ which has the same integral homology as $S^{k} \times S^{n-k}$, then Cecil and Ryan proved in [CR78] that $M$ has precisely two principal curvatures at each point and that the principal curvatures are constant along the corresponding curvature distributions.

Bott and Samelson's construction of an explicit $\mathbf{Z}_{2}$-homology basis for orbits of variationally complete representations can now be rephrased: the orbits of variationally complete representations are taut submanifolds. Recall that, by the results of [DO01, Dad85], these are precisely the orbits of the isotropy representations of the symmetric spaces, the so called generalized flag manifolds.

The generalized flag manifolds are homogeneous examples of submanifolds which belong to another very important, more general class of submanifolds, called isoparametric submanifolds. An isoparametric hypersurface in a simplyconnected real space form is a hypersurface with constant principal curvatures (see [Tho00] for an extensive survey on isoparametric hypersurfaces and their generalizations). In the course of his work on the subject, Cartan noticed that isoparametric hypersurfaces in spheres is a much more rich and difficult object of study than its counterparts in Euclidean and hyperbolic spaces. In fact, until today there is no complete classification of them. The subject seems to have been forgotten for over thirty years after Cartan, when Münzner (see also [Nom73]) wrote the two very influential papers [Mue80, Mue81]. Using his results, Cecil and Ryan observed in [CR81] that isoparametric hypersurfaces and their focal manifolds are taut.

In the eighties, some generalizations of the concept of isoparametric hypersurface to higher codimensions were proposed, but the today commonly accepted one seems to have been first given by Harle in [Har82] (see also Carter and West [CW85a, CW85b] and Terng [Ter85]). An isoparametric submani- 
fold of a simply-connected space form is a submanifold whose normal bundle is flat and such that, for every locally defined parallel normal vector field, the eigenvalues of the corresponding Weingarten operator are constant. Palais and Terng [PT87] showed that a compact homogeneous isoparametric submanifold of Euclidean space is a principal orbit of a polar representations, and then it follows from Dadok's theorem that it must be a generalized flag manifold. Examples of inhomogeneous isoparametric hypersurfaces in spheres were constructed in [OT75, OT76] and, more systematically, in [FKM81]. In contrast, Thorbergsson proved in [Tho91] the striking result that a compact irreducible isoparametric submanifold of substantial codimension greater than 2 in an Euclidean space is homogeneous (see [Olm93, HL99, Esc00] for other proofs of this fact), and then it follows as above that it must be a generalized flag manifold. Hsiang, Palais and Terng studied in [HPT88] the topology of isoparametric submanifolds and proved, among other things, that they and their focal submanifolds are taut. This result also follows from the work of Thorbergsson in [Tho83]. Both in [HPT88] and [Tho83], the method to prove tautness of a submanifold is to construct explicit cycles representing the $\mathbf{Z}_{2}$-homology as maps of iterated bundles of curvature surfaces (generically, spheres) associated to the focal points along geodesics normal to that submanifold, which can be viewed as a generalization of the method of Bott and Samelson to show that the generalized flag manifolds are taut.

Most of the examples of taut embeddings known are homogeneous spaces. In [Tho88] Thorbergsson posed some questions regarding the problem of which homogeneous spaces admit taut embeddings and derived some necessary topological conditions for the existence of a taut embedding which allowed him to conclude that certain homogeneous spaces cannot be tautly embedded (see also [Heb88]), among others the lens spaces distinct from the real projective space. Olmos showed in [Olm94] that a compact homogeneous submanifold embedded in Euclidean space with a flat normal bundle is a generalized flag manifold. Many proofs have been given of the tautness of special cases of generalized flag manifolds where the arguments are easier. No new examples of 
taut embeddings of homogeneous spaces besides the generalized flag manifolds were known until Thorbergsson and I classified in [GT03] (see also [GT00]) the irreducible representations of compact Lie groups all of whose orbits are tautly embedded; we call these representations taut. It turns out that the classification includes three new families of representations which are not isotropy representations of symmetric spaces, thereby supplying many new examples of $\left(\mathbf{Z}_{2^{-}}\right)$ tautly embedded homogeneous spaces, namely $(n \geq 2)$ :

\begin{tabular}{|c|c|}
\hline $\mathbf{S O}(2) \times \mathbf{S p i n}(9)$ & (standard) $\otimes_{\mathbf{R}}($ spin $)$ \\
$\mathbf{U}(2) \times \mathbf{S p}(n)$ & $($ standard $) \otimes_{\mathbf{C}}($ standard $)$ \\
$\mathbf{S U}(2) \times \mathbf{S p}(n)$ & $(\text { standard })^{3} \otimes_{\mathbf{H}}($ standard $)$ \\
\hline
\end{tabular}

It is interesting to remark that these three families of representations precisely coincide with the representations of cohomogeneity 3 of the compact Lie groups which are not orbit equivalent to the isotropy representation of a symmetric space.

The proof of this classification result is long and involved. It starts with Kuiper's observation that was mentioned in the last paragraph of section 4 . It implies that a taut irreducible representation has the property that the second osculating spaces of any of its nontrivial orbits coincide with the ambient space; we say that representations with this property are of class $\mathcal{O}^{2}$. The class $\mathcal{O}^{2}$ is much more tractable than the class of taut representations since it involves an infinitesimal condition. The technique of Dadok [Dad85], notably his invariant $k(\lambda)$, turns out to be an extremely powerful tool to reduce the class $\mathcal{O}^{2}$ in size so that the remaining cases are amenable to the geometric methods that we develop in the second part of the proof.

In [GT02a] Thorbergsson and I provided another proof of the $\mathbf{Z}_{2}$-tautness of the orbits of the three families of exceptional taut irreducible representations by adapting the construction of the cycles of Bott and Samelson to that case. The main new technical difficulty that we encountered was that focal points in the direction of normal vector fields parallel along some curvature circles do not have constant multiplicity, which made a modification in the construction of 
the cycles necessary to prevent the bundles from having some degenerate fibers.

Recently, I completed the classification of taut reducible representations of compact simple Lie groups [Gor04b]. The list is

\begin{tabular}{|c|c|c|}
\hline $\mathbf{S U}(n), n \geq 3$ & $\mathbf{C}^{n} \oplus \cdots \oplus \mathbf{C}^{n}$ & $k$ copies, where $1<k<n$ \\
\hline $\mathbf{S O}(n), n \geq 3, n \neq 4$ & $\mathbf{R}^{n} \oplus \cdots \oplus \mathbf{R}^{n}$ & $k$ copies, where $1<k$ \\
\hline $\mathbf{S p}(n), n \geq 1$ & $\mathbf{C}^{2 n} \oplus \cdots \oplus \mathbf{C}^{2 n}$ & $k$ copies, where $1<k$ \\
\hline $\mathbf{G}_{2}$ & $\mathbf{R}^{7} \oplus \mathbf{R}^{7}$ & - \\
\hline $\mathbf{S p i n}(6)$ & $\mathbf{R}^{6} \oplus \mathbf{C}^{4}$ & $\mathbf{R}^{6}=$ (vector), $\mathbf{C}^{4}=($ spin $)$ \\
\hline $\operatorname{Spin}(7)$ & $\mathbf{R}^{7} \oplus \mathbf{R}^{8}$ & \\
& $\mathbf{R}^{8} \oplus \mathbf{R}^{8}$ & $\mathbf{R}^{7}=$ (vector), $\mathbf{R}^{8}=($ spin $)$ \\
& $\mathbf{R}^{8} \oplus \mathbf{R}^{8} \oplus \mathbf{R}^{8}$ & \\
\hline & $\mathbf{R}^{7} \oplus \mathbf{R}^{7} \oplus \mathbf{R}^{8}$ & \\
\hline $\operatorname{Spin}(8)$ & $\mathbf{R}_{0}^{8} \oplus \mathbf{R}_{+}^{8}$ & \\
& $\mathbf{R}_{0}^{8} \oplus \mathbf{R}_{0}^{8} \oplus \mathbf{R}_{+}^{8}$ & $\mathbf{R}_{0}^{8}=($ vector$), \mathbf{R}_{+}^{8}=($ halfspin$)$ \\
\hline $\operatorname{Spin}(9)$ & $\mathbf{R}_{0}^{8} \oplus \mathbf{R}_{0}^{8} \oplus \mathbf{R}_{0}^{8} \oplus \mathbf{R}_{+}^{8}$ & \\
\hline & $\mathbf{R}^{16} \oplus \mathbf{R}^{16}$ & $\mathbf{R}^{16}=($ spin $)$ \\
\hline
\end{tabular}

Despite these results, the classification of taut homogeneous submanifolds of Euclidean spaces is still far from complete. We still do not even know what the reducible taut representations of the nonsimple compact Lie groups are.

\section{The geometry of the exceptional taut irre- ducible representations and copolarity of iso- metric actions}

The principal orbits of the exceptional taut irreducible representations fail to be isoparametric because focal points in the direction of normal vector fields parallel along some curvature circles do not have constant multiplicity. From another viewpoint, the distribution of normal spaces to the principal orbits along a normal geodesic fails to be constant just because of a one-dimensional subspace. As a matter of fact, the construction of the cycles in [GT02a] relied on a detailed knowledge of the geometry of the orbits of those representations. This posed the question of whether it was possible to generalize the geometry of those representations.

It turned out that Olmos, Tojeiro and I defined in [GOT04] a minimal $k$ - 
section through a regular point of an isometric action of a compact Lie group $G$ on a complete Riemannian manifold $M$ as being the smallest connected, complete, totally geodesic submanifold of $M$ through that point which intersects all the orbits and such that, at any intersection point with a principal orbit, its tangent space contains the normal space of that orbit with codimension $k$. This is a good definition and uniquely specifies an integer $k$ which we call the copolarity of the isometric action. It is also obvious that the $k=0$ case precisely corresponds to the polar actions.

It is apparent that for most isometric actions the minimal $k$-section coincides with the ambient space. Note that in this case $k$ equals the dimension of a principal orbit. We say that such isometric actions have trivial copolarity. The obvious questions that emerge are:

What are the isometric actions with nontrivial copolarity? What is the meaning of the integer $k$ ?

In [GOT04] we examined this problem in the case of orthogonal representations, and we gave a complete answer to the above questions for the extremal values of the invariant $k$. First consider the case $k=1$. An irreducible representation of nontrivial copolarity $k=1$ is one of the following orthogonal representations $(n \geq 2)$ :

\begin{tabular}{|c|c|}
\hline $\mathbf{S O}(2) \times \mathbf{S p i n}(9)$ & (standard) $\otimes_{\mathbf{R}}($ spin $)$ \\
$\mathbf{U}(2) \times \mathbf{S p}(n)$ & $($ standard $) \otimes_{\mathbf{C}}($ standard $)$ \\
$\mathbf{S U}(2) \times \mathbf{S p}(n)$ & $(\text { standard })^{3} \otimes_{\mathbf{H}}($ standard $)$ \\
\hline
\end{tabular}

In view of the above discussion of taut irreducible representations, we are lead to the beautiful characterization of taut irreducible representations as those irreducible representations admitting $k=0$ or $k=1$.

The proof of the preceeding classification result is based on a geometric description of the principal orbits of (not necessarily irreducible) representations with copolarity $k=1$ obtained in [GOT04]. Namely, it was shown that such an orbit splits extrinsically as a product of a homogeneous isoparametric 
submanifold (which can happen to be just a point) and another homogeneous submanifold which is either:

- a nonisoparametric curve;

- a focal manifold of a homogeneous irreducible isoparametric submanifold which is obtained by focalizing a one-dimensional distribution; or

- a codimension 3 nonisoparametric submanifold.

The main tools to obtain this description are the results of Olmos on the normal holonomy of submanifolds [Olm90, Olm93, Olm94, OS95], that is, the holonomy of the connection induced on the normal bundle, and the main result of [GT03].

Finally, in regard to large values of $k$, we mention that it was shown in [GOT04] that the codimension of a nontrivial minimal $k$-section of a nonpolar irreducible representation is at least 3 .

\section{Concluding remarks}

The geometry of the orbits of isometric actions on complete Riemannian manifolds is a classical, yet still fascinating subject. At the cornerstone of this theory are situated the generalized flag manifolds. They represent to submanifold theory what the Riemannian symmetric spaces represent to intrisic Riemannian geometry. At the same time, the former are the principal orbits of the linear isotropy representations of the latter. The generalized flag manifolds constitute model spaces in several areas of geometry, topology and representation theory. Their submanifold properties are abstracted in the concept of an isoparametric submanifold, and their representation-theoretic ones in the concept of a polar representation. Moreover, from the Morse-theoretic point of view, they constitute the main body of examples of taut submanifolds of Euclidean space. Their nice properties have been weakened in the form of the consideration of other broader, classes of submanifolds (e.g. Dupin submanifolds [Pin85, Pin86], weakly isoparametric submanifolds [Ter87]), or 
generalized to accomodate other ambient spaces (e.g. polar actions on Hilbert

spaces [Ter95], taut submanifolds of Hilbert spaces [TT95], equifocal and taut submanifolds of symmetric spaces [TT95, TT97], isoparametric submanifolds of general ambient spaces [HLO00]), or viewed from the point of view of singular foliations (singular Riemannian foliations with sections [Ale04], submanifolds with parallel focal structure [Toe04]), to name a few of the ramifications and connections. The lines of investigation in the near future will probably focus on pushing further the results about actions on symmetric spaces and infinite dimensional Hilbert spaces. We also mention that the case of actions of noncompact Lie groups is still rather poorly understood. Another link is provided by the complexification of the compact Lie groups, the so called complex reductive linear algebraic groups, acting on complex vector spaces and complex manifolds, as has already been pointed out in [HW90, PT02, PT03].

\section{References}

[Ale04] Alexandrino, M. M., Singular Riemannian foliations with sections, Illinois J. Math. 48 (4) (2004), 1163-1182.

[Ban65] Banchoff, T. F., Tightly embedded 2-dimensional polyhedral manifolds, Amer. J. Math. 87 (1965), 462-472.

[Ban70] _ The spherical two-piece property and tight surfaces in spheres, J. Differential Geom. 4 (1970), 193-205.

[BCO03] Berndt, J., Console, S., Olmos, C., Submanifolds and holonomy, Research Notes in Math. 434, Chapman \& Hall/CRC, Boca Raton, 2003.

[Ber01] Bergmann, I., Reducible polar representations, Manuscripta Math. 104 (2001), no. 3, 309-324.

[BG05] Biliotti, L., Gori, A., Coisotropic and polar actions on complex Grassmannians, Trans. Amer. Math. Soc. 357 (5) (2005), 1731-1751. 
[Bil04] Biliotti, L., Coisotropic and polar actions on compact irreducible Hermitian symmetric spaces, E-print math. DG/0408409, 2004.

[Bot56] Bott, R., An application of the Morse theory to the topology of Lie groups, Bull. Soc. Math. France 84 (1956), 251-281.

[Bou95] Boualem, H., Feuilletages riemanniens singuliers transversalement intégrables, Compositio Math. 95 (1995), no. 1, 101-125.

[BS58] Bott, R., Samelson, H., Applications of the theory of Morse to symmetric spaces, Amer. J. Math. 80 (1958), 964-1029, Correction in Amer. J. Math. 83 (1961), 207-208.

[Cec97] Cecil, T. E., Taut and Dupin submanifolds, Tight and Taut Submanifolds (T. E. Cecil and S.-S. Chern, eds.), Math. Sci. Res. Inst. Publ. 32, Cambridge University Press, (1997), 135-180.

[CL57] Chern, S. S., Lashof, R., On the total curvature of immersed manifolds, Amer. J. Math. 79 (1957), 306-318.

[Con71] Conlon, L., Variational completeness and K-transversal domains, J. Differential Geom. 5 (1971), 135-147.

[CR78] Cecil, T. E., Ryan, P. J., Focal sets, taut embeddings and the cyclides of Dupin, Math. Ann. 236 (1978), 177-190.

[CR81] _ Tight spherical embeddings, Lecture Notes in Math., no. 838, Springer-Verlag, (1981), 94-104.

[CR85] — Tight and taut immersions of manifolds, Research Notes in Mathematics, no. 107, Pitman, (1985).

[CW72] Carter, S., West, A., Tight and taut immersions, Proc. London. Math. Soc. 25 (1972), 701-720.

[CW85a] _ Generalised Cartan polynomials, J. London. Math. Soc. 32 (1985), 305-316. 
[CW85b] _ Isoparametric systems and transnormality, Proc. London. Math. Soc. 51 (1985), 520-542.

[Dad85] Dadok, J., Polar actions induced by actions of compact Lie groups, Trans. Amer. Math. Soc. 288 (1985), 125-137.

[DO01] Di Scala, A. J., Olmos, C., Variationally complete representations are polar, Proc. Amer. Math. Soc. 129 (2001), 3445-3446.

[EH99] Eschenburg, J., Heintze, E., On the classification of polar representations, Math. Z. 232 (1999), 391-398.

[Esc00] Eschenburg, J., Isoparametric submanifolds and symmetric spaces, Geometry and Topology: Aarhus (1998), Contemp. Math., vol. 258, Amer. Math. Soc., (2000), 145-155.

[FKM81] Ferus, D., Karcher, H., Muenzner, H. F., Cliffordalgebren and neue isoparametrische Hyperflaechen, Math. Z. 177 (1981), 479-502.

[Gor01] Gorodski, C., Tightness and tautness, an Appendix to "Notes on Morse theory", by D. Tausk, F. Mercuri and P. Piccione, Publicações Matemáticas do IMPA, 23 Colóquio Brasileiro de Matemática, IMPA, 2001.

[Gor04a] _ Polar actions on compact symmetric spaces which admit a totally geodesic principal orbit, Geom. Dedicata 103 (2004), 193-204.

[Gor04b] _ , Taut reducible representations of compact simple Lie groups, E-print math. DG/0404269, 2004.

[GOT04] Gorodski, C., Olmos, C., Tojeiro, R., Copolarity of isometric actions, Trans. Amer. Math. Soc. 356 (2004), 1585-1608.

[GT00] Gorodski, C., Thorbergsson, G., Representations of compact Lie groups and the osculating spaces of their orbits, Preprint, Univ. of Cologne, (also E-print math. DG/0203196), 2000. 
[GT02a] - Cycles of Bott-Samelson type for taut representations, Ann. Global Anal. Geom. 21 (2002), 287-302.

[GT02b] - Variationally complete action on compact symmetric spaces, J. Differential Geom. 62 (2002), 39-48.

[GT03] - The classification of taut irreducible representations, J. Reine Angew. Math. 555 (2003), 187-235.

[Har82] Harle, C. E., Isoparametric families of submanifolds, Bol. Soc. Brasil. Mat. 13 (1982), 35-48.

[Heb88] Hebda, J., The possible cohomology ring of certain types of taut submanifolds, Nagoya Math. J. 111 (1988), 85-97.

[Her60] Hermann, R., Variational completeness for compact symmetric spaces, Proc. Amer. Math. Soc. 11 (1960), 544-546.

[HL99] Heintze, E., Liu, X., Homogeneity of infinite-dimensional isoparametric submanifolds, Ann. Math. 149 (1999), 149-181.

[HLO00] Heintze, E., Liu, X., Olmos, C., Isoparametric submanifolds and a Chevalley-type restriction theorem, E-print math. DG/0004028, 2000.

[HPT88] Hsiang, W.-Y., Palais, R. S. Terng, C.-L., The topology of isoparametric submanifolds, J. Differential Geom. 27 (1988), 423-460.

[HW90] Huckleberry, A. T., Wurzbacher, T., Multiplicity-free complex manifolds, Math. Ann. 286 (1990), 261-280.

[Kol02] Kollross, A., A classification of hyperpolar and cohomogeneity one actions, Trans. Amer. Math. Soc. 354 (2002), 571-612.

[Kui58] Kuiper, N. H., Immersions with minimal total absolute curvature, Coll. de géométrie diff., Centre Belge de Recherches Math., Bruxelles, (1958), 75-88. 
[Kui61] _ Sur les immersions à courbure totale minimale, Séminaire de Topologie et Géometrie Différentielle C. Ereshmann, Paris, vol. II, 1961, Recueil d'exposés faits en 1958-1959-1960.

[Kui62] — On convex maps, Nieuw Archief voor Wisk. 10 (1962), 147164.

[Mue80] Muenzner, H. F., Isoparametrische Hyperflaechen in Sphaeren, I, Math. Ann. 251 (1980), 57-71.

[Mue81] _ Isoparametrische Hyperflaechen in Sphaeren, II, Math. Ann. 256 (1981), 215-232.

[Nom73] Nomizu, K., Some results in É. Cartan's theory of isoparametric families of hypersurfaces, Bull. Amer. Math. Soc. 79 (1973), 1184-1188.

[Olm90] Olmos, C., The normal holonomy group, Proc. Amer. Math. Soc. 110 (1990), 813-818.

[Olm93] _ Isoparametric submanifolds and their homogeneous structures, J. Differential Geom. 38 (1993), 225-234.

[Olm94] - Homogeneous submanifolds of higher rank and parallel mean curvature, J. Differential Geom. 39 (1994), 605-627.

[OS95] Olmos, C., Salvai, M., Holonomy of homogeneous vector bundles and polar representations, Indiana Univ. Math. J. 44 (1995), 1007-1015.

[OT75] Ozeki, H., Takeuchi, M., On some types of isoparametric hypersurfaces in spheres, I, Tôhoku Math. J. 27 (1975), 515-559.

[OT76] - On some types of isoparametric hypersurfaces in spheres, II, Tôhoku Math. J. 28 (1976), 7-55.

[Pin85] Pinkall, U., Dupin hypersurfaces, Math. Ann. 270 (1985), 427-440. 
[Pin86] - Curvature properties of taut submanifolds, Geom. Dedicata 20 (1986), 79-83.

[PT87] Palais, R. S., Terng, C.-L., A general theory of canonical forms, Trans. Amer. Math. Soc. 300 (1987), 771-789.

[PT99] Podestà, F., Thorbergsson, G., Polar actions on rank one symmetric spaces, J. Differential Geom. 53 (1999), 131-175.

[PT02] - Polar and coisotropic actions on Kähler manifolds, Trans. Amer. Math. Soc. 354 (2002), 1759-1781.

[PT03] - Coisotropic actions on compact homogeneous Kähler manifolds, Math. Z. 243, no. 3, (2003), 471-490.

[Ter85] Terng, C.-L., Isoparametric submanifolds and their Coxeter groups, J. Differential Geom. 21 (1985), 79-107.

[Ter87] - Submanifolds with flat normal bundle, Math. Ann. 277, no. 1, (1987), 95-111.

[Ter95] — Polar actions on Hilbert space, J. Geom. Anal. 5, no. 1, (1995), 129-150.

[Tho83] Thorbergsson, G., Dupin hypersurfaces, Bull. London Math. Soc. 15 (1983), 493-498.

[Tho88] - Homogeneous spaces without taut embeddings, Duke Math. J. 57 (1988), 347-355.

[Tho91] _ Isoparametric foliations and their buildings, Ann. of Math. (2) 133 (1991), 429-446.

[Tho98] — Smooth tight immersions, Jber. d. Dt. Math.-Verein. 100 (1998), 23-35. 
[Tho00] _ A survey on isoparametric hypersurfaces and their generalizations, Handbook of Differential Geometry, vol. I, ch. 10, Elsevier Science, 2000.

[Toe04] Toeben, D., Parallel focal structure and singular Riemannian foliations, E-print math. DG/0403050, 2004.

[TT95] Terng, C. L., Thorbergsson, G., Submanifold geometry in symmetric spaces, J. Differential Geom 42, no. 3, (1995), 665-718.

[TT97] _ Taut immersions into complete Riemannian manifolds, Tight and Taut Submanifolds (T. E. Cecil and S.-S. Chern, eds.), Math. Sci. Res. Inst. Publ. 32, Cambridge University Press, (1997), 181-228.

Instituto de Matemática e Estatística

Universidade de São Paulo

Rua do Matão, 1010

05508-090, São Paulo, SP, Brasil

e-mail: gorodski@ime.usp.br 